\title{
Mapping between Hamiltonians with attractive and repulsive potentials on a lattice
}

\author{
Yogesh N. Joglekar \\ Department of Physics, Indiana University Purdue University \\ Indianapolis (IUPUI), Indianapolis, Indiana 46202, USA
}

(Dated: October 15, 2018)

\begin{abstract}
Through a simple and exact analytical derivation, we show that for a particle on a lattice, there is a one-to-one correspondence between the spectra in the presence of an attractive potential $\hat{V}$ and its repulsive counterpart $-\hat{V}$. For a Hermitian potential, this result implies that the number of localized states is the same in both, attractive and repulsive, cases although these states occur above (below) the band-continnum for the repulsive (attractive) case. For a $\mathcal{P} \mathcal{T}$-symmetric potential that is odd under parity, our result implies that in the $\mathcal{P} \mathcal{T}$-unbroken phase, the energy eigenvalues are symmetric around zero, and that the corresponding eigenfunctions are closely related to each other.
\end{abstract}


Introduction: The energy spectrum of a quantum particle in an attractive potential $V(\mathbf{r})$, in general, consists of discrete eigenvalues for which the eigenfunctions are localized in real space, and continuum eigenvalues with non square-integrable eigenfunctions. The energy spectrum for the corresponding repulsive potential $-V(\mathbf{r})$ has only continuum eigenvalues [1, 2]. This situation changes dramatically when the particle is confined to a lattice or, equivalently, is exposed to a periodic potential. Indeed, repulsively bound two-atom states have been explored in detail since their experimental discovery in optical lattices [3, 4] and continue to be a source of ongoing work [5] in the context of the Bose-Hubbard model [6, 7]. We note that in the Bose-Hubbard model, the interaction between the two atoms is shortranged and is tuned via the Feschback resonance [3]. However, to our knowledge, the properties of single-particle states localized in the vicinity of a generic repulsive potential (defined below) have not been studied. In another area, localized states in parity + timereversal $(\mathcal{P} \mathcal{T})$ symmetric one-dimensional lattice models, too, have been explored in recent years. These explorations have focused on the $\mathcal{P} \mathcal{T}$-symmetry breaking in the presence of attractive (real) on-site potentials with random $\mathcal{P} \mathcal{T}$-symmetric complex parts [11].

In this note, through a simple but exact derivation, we show that for a single particle on a lattice, there is a one-to-one correspondence between its energy spectrum in the presence of an attractive potential and the repulsive counterpart, and that the corresponding eigenfunctions have identical probability distributions. For $\mathcal{P} \mathcal{T}$-symmetric potentials that are odd under parity (and hence time-reversal), we show that if the $\mathcal{P} \mathcal{T}$-symmetry is unbroken, the energy spectrum must be symmetric around zero.

One-dimensional Model: Let us start with the Hamiltonian for a particle on a onedimensional lattice with only nearest-neighbor hopping energy $J>0$,

$$
\hat{H}_{0}=-J \sum_{i}\left(c_{i}^{\dagger} c_{i+1}+c_{i+1}^{\dagger} c_{i}\right)
$$

where $c_{i}^{\dagger}$ and $c_{i}$ are creation and annihilation operators at site $i$ respectively. The external potential is given by $\hat{V}=\sum_{j} V_{j} c_{j}^{\dagger} c_{j}$. We define the potential to be attractive provided $\sum_{j} V_{j}<0$ and repulsive if is positive. Let $\left|\psi_{\alpha}\right\rangle=\sum_{j} f_{\alpha, j}|j\rangle$ be an eigenstate of the Hamiltonian $\hat{H}_{+}=\hat{H}_{0}+\hat{V}$ with energy $E_{\alpha}$ where $|j\rangle$ denotes a single-particle state localized at site $j$. The coefficients $f_{\alpha, j}$ obey the recursion relation

$$
-J\left[f_{\alpha, j+1}+f_{\alpha, j-1}\right]+V_{j} f_{\alpha, j}=E_{\alpha} f_{\alpha, j}
$$


We now consider the staggered wavefunction $\left|\phi_{\alpha}\right\rangle=\sum_{j} f_{\alpha, j}(-1)^{j}|j\rangle$. Using Eq.(2) it is straightforward to show that the staggered wavefunction satisfies the following equation

$$
\hat{H}_{0}\left|\phi_{\alpha}\right\rangle=\left(-E_{\alpha}+\hat{V}\right)\left|\phi_{\alpha}\right\rangle
$$

Thus, it is an eigenfunction of the conjugate Hamiltonian $\hat{H}_{-}=\hat{H}_{0}-\hat{V}$ with eigenvalue $-E_{\alpha}$. When $\hat{V}=0$, the energy spectrum is given by $\epsilon_{k}=-2 J \cos (k a)$ and represents the well-known continuum band from $-2 J$ to $2 J$ where $a$ is the lattice spacing. In this trivial case, indeed the eigenfunction $\left|\psi_{k}\right\rangle=\sum_{j} \sin (k j)|j\rangle$ and its staggered counterpart $\left|\phi_{k}\right\rangle=\sum_{j} \sin [(\pi-k) j]|j\rangle$ have energies $\pm \epsilon_{k}$ respectively.

Our result shows that if an attractive external potential $\hat{V}$ has $n$ bound states below its continuum with energies $E_{m}(m=1, \ldots, n)$, then the corresponding repulsive potential $-\hat{V}$ must have an equal number of bound states above its continuum with energies $-E_{m}$. Since the staggered wavefunction $\left|\phi_{\alpha}\right\rangle$ varies over the lattice length-scale $a$, it is energetically expensive and ill-defined in the continuum limit $a \rightarrow 0$. Physically, in the continuum limit, the absence of lattice-site scattering centers makes it impossible for a particle to localize near the repulsive potential. However, on a lattice, the probability distributions for the two states - a localized bound state $\left|\psi_{\alpha}\right\rangle$ with energy $E_{\alpha} \leq-2 J$ in an attractive potential and the localized bound state $\left|\phi_{\alpha}\right\rangle$ with energy $-E_{\alpha} \geq+2 J$ in the repulsive potential - are identical. As a concrete example, we numerically obtain the spectrum for a lattice with $N=29$ sites and a quadratic potential that vanishes at the ends, $V_{m}=\Lambda(m-1)(N-m) / N_{0}^{2}$, where $m=1, \ldots, N, N_{0}=(N+1) / 2$ is the center of the lattice and $V_{N_{0}}=\Lambda$. Figure 1 1 shows the ground state wavefunction $\psi_{G m}$ for the attractive case, $\Lambda / J=-0.5$, (left panel) along with the highest-energy state wavefunction $\phi_{m}$ for the repulsive case, $\Lambda / J=+0.5$ (right panel). It is clear that the two wavefunctions are related by $\phi_{m}=(-1)^{m+1} \psi_{G, m}$.

Two-particle Case: We can generalize this result in a straightforward manner to treat interparticle interaction $\hat{U}=\sum_{i j} U_{i-j} \hat{n}_{i} \hat{n}_{j}$ where the on-site number operator is given by $\hat{n}_{i}=c_{i}^{\dagger} c_{i}$. In the two-particle sector, the recursion relation satisfied by the relative-coordinate wavefunction is given by [6, 7]

$$
-J_{K}\left[\psi_{\alpha, m+1}^{K}+\psi_{\alpha, m-1}^{K}\right]+U\left(r_{m}\right) \psi_{\alpha, m}^{K}=E_{\alpha}^{K} \psi_{\alpha, m}^{K} .
$$

Here $-\pi / a \leq K \leq \pi / a$ is the lattice momentum associated with the center-of-mass of the two particles, $J_{K}=J \cos (K a)$ is the effective hopping energy, $r_{m}=a m=a(i-j)$ is the 

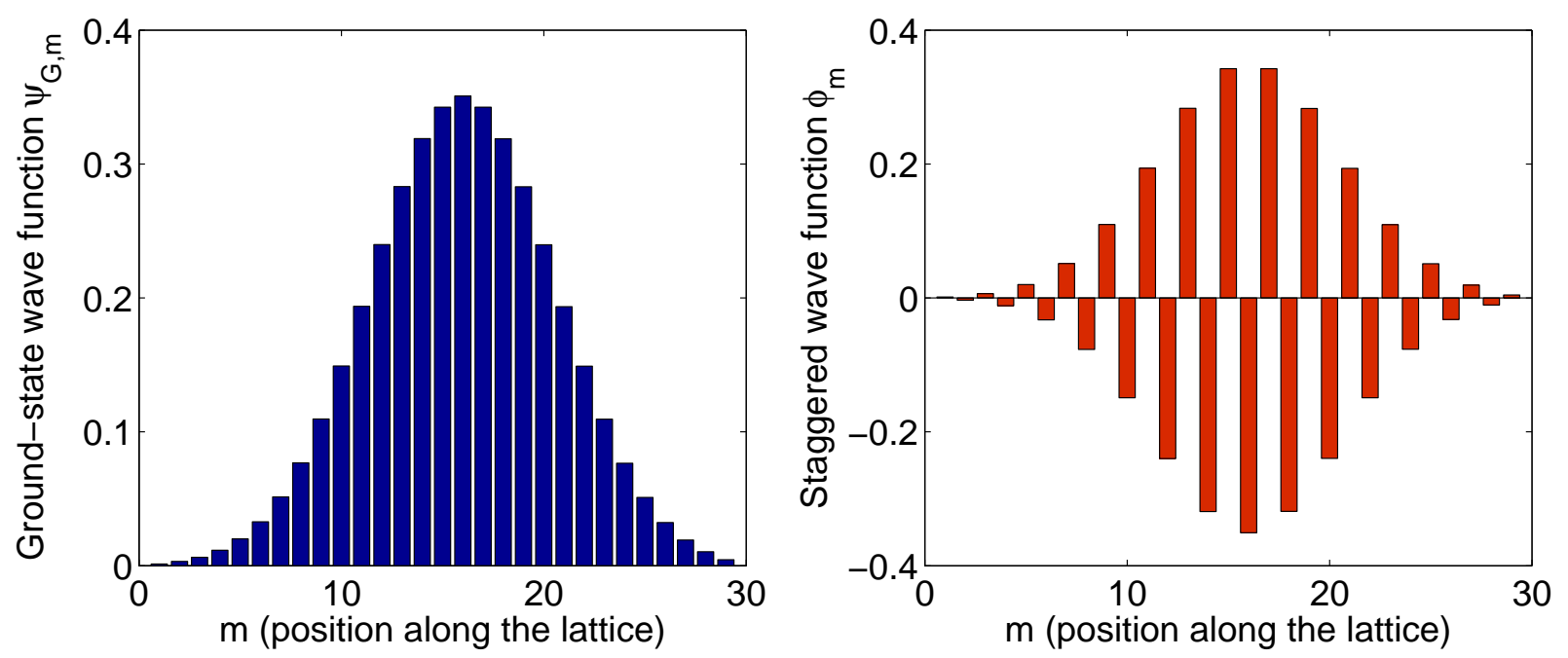

FIG. 1. (color online) (a) The left panel shows the dimensionless ground-state wavefunction $\psi_{G, m}$ for an attractive quadratic potential $V_{m}=\Lambda(m-1)(N-m) / N_{0}^{2}$ where $N=29=\left(2 N_{0}+1\right)$ is the lattice size and $\Lambda / J=-0.5$. As expected for a quadratic potential ground-state, $\psi_{G, m}$ is a Gaussian with width $x_{0}=a\left(N_{0}^{2} t /|\Lambda|\right)^{1 / 4} \sim 4$.61. (b) The right panel shows the dimensionless highest-energy state wavefunction $\phi_{m}$ for its repulsive counterpart with $\Lambda / J=+0.5$. We see that the $\phi_{m}$ is indeed the staggered version of the ground-state wavefunction $\phi_{G, m}$.

distance between the two particles on the lattice located at sites $i$ and $j$, and $U\left(r_{m}\right)$ is the real-space interaction between the two particles. Note that for a non-local interparticle interaction $U\left(r_{m}\right)$, multiple bound-state $\psi_{\alpha}^{K}$ solutions are generic, although, in the context of the Bose-Hubbard model, only one [3] or two [6] have been discussed. If $\psi_{\alpha}^{K}$ is an eigenfunction of the Hamiltonian $\hat{H}_{0}+\hat{U}$ with energy $E_{\alpha}^{K}$, Eq. 4 implies that the staggered wavefunction $\phi_{\alpha}^{K}$ defined by $\phi_{\alpha}^{K}\left(r_{m}\right)=(-1)^{m} \psi_{\alpha}^{K}\left(r_{m}\right)$ is an eigenfunction of the conjugate Hamiltonian $\hat{H}_{0}-\hat{U}$ with energy $-E_{\alpha}^{K}$.

Two-particle bound states in the presence of on-site and nearest-neighbor repulsive density-density interactions on a lattice have been extensively investigated [3, 5, 6]. Our derivation shows that they are a generic feature of any density-density interaction on a lattice, and this result is true for square lattices in higher dimensions. Note that the quantum statistics of the particles only constrains the relative wavefunction $\psi_{\alpha}^{K}\left(r_{m}\right)$ to be odd (spinless fermions) or even (bosons or spin-singlet fermions) under parity; however, it does not 
affect the one-to-one correspondence between the spectra for the two Hamiltonians $\hat{H}_{0} \pm \hat{U}$. Thus, two-atom bound-states with attractive and repulsive interactions in optical lattices (bosons) [3], the donor and acceptor impurity levels in semiconductors (fermions) [8], as well as the localized phonon modes (collective bosonic excitation) [9, 10] around a soft or stiff impurity can all be thought of as manifestations of the correspondence between spectra for $\hat{H}_{+}$and $H_{-}$.

$\mathcal{P} \mathcal{T}$ Symmetric Potential: The mapping between the two Hamiltonians $\hat{H}_{+}$and $\hat{H}_{-}$is valid independent of the properties of the potential $\hat{V}$ including its Hermiticity; the on-site potential elements $V_{j}$ may be complex. However, for a $\mathcal{P} \mathcal{T}$-symmetric potential that is odd under parity (and hence, time reversal), $V_{j}^{*}=-V_{j}=V_{-j}$, it follows that $\hat{H}_{+}^{*}=\hat{H}_{-}$where * denotes complex conjugation. Therefore, it follows from $\hat{H}_{+}\left|\psi_{\alpha}\right\rangle=E_{\alpha}\left|\psi_{\alpha}\right\rangle$ that the wavefunction $\left|\psi_{\alpha}^{*}\right\rangle=\sum_{j} f_{\alpha, j}^{*}|j\rangle$ is an eigenstate of the conjugate Hamiltonian $\hat{H}_{-}$with eigenvalue $+E_{\alpha}^{*}$. In the continuum limit, it has been shown that a wide class of such potentials, including $V(x)=i x^{3}$ and $V(x)=i \sin ^{2 n+1}(x)$ have purely real energy spectra [12, 13]. If the $\mathcal{P} \mathcal{T}$-symmetry is unbroken, $E_{\alpha}^{*}=E_{\alpha}$, then it follows that $\hat{H}_{-}\left|\phi_{\alpha}\right\rangle=-E_{\alpha}\left|\phi_{\alpha}\right\rangle$ and $\hat{H}_{-}\left|\psi_{\alpha}^{*}\right\rangle=+E_{\alpha}\left|\psi_{\alpha}^{*}\right\rangle$.

This explicit construction of wavefunctions with equal and opposite energies implies that for any arbitrary $\mathcal{P} \mathcal{T}$-symmetric potential that is odd under parity, if the $\mathcal{P} \mathcal{T}$ symmetry is not broken, the energy spectrum must be symmetric around zero. It also shows that the corresponding wavefunctions in the two cases have components that are simply related: $\left[+E_{\alpha}, f_{\alpha, j}^{*}\right] \leftrightarrow\left[-E_{\alpha}, f_{\alpha, j}(-1)^{j}\right]$. As an example, we consider the simplest "finite lattice" with 2 points. (Our result is equally applicable to a finite lattice.) The Hamiltonian in this case is given by $\hat{H}_{-}=-J \hat{\sigma}_{x}+i \gamma \hat{\sigma}_{z}$ where $\left(\sigma_{x}, \sigma_{z}\right)$ are the Pauli matrices in the site-index space [14] and a real $\gamma$ ensures that the potential is odd under parity as well as time-reversal. The eigenvalues in this case are given by $E_{ \pm}= \pm \sqrt{J^{2}-\gamma^{2}}$. Thus the $\mathcal{P} \mathcal{T}$-symmetry in this case is not broken as long as $\gamma \leq J$. The corresponding (unnormalized) eigenfunctions [15] are given by [14]

$$
| \pm\rangle=\left(\begin{array}{c}
1 \\
\pm e^{\mp i \theta}
\end{array}\right)
$$

where $\theta=\arctan \left(\gamma / \sqrt{J^{2}-\gamma^{2}}\right)$ is real when $\gamma \leq J$. Therefore, in the $\mathcal{P} \mathcal{T}$-unbroken phase, the eigenvectors for positive and negative energies indeed are related by $f_{-, j}=(-1)^{j} f_{+, j}^{*}$ where $j=0,1$. 
Conclusion: Our result, through a one-to-one mapping between attractive and repulsive potentials on a lattice, shows that localized states in repulsive potentials are ubiquitous. These states can be explored via local measurements. In contrast to the bound-states with energies below the continuum band, these localized states with energies above the continuum band will decay into the continnum states. They may thus provide a useful spectroscopic tool in optical lattices as well as engineered electronic materials with a small bandwidth.

[1] L.D. Landau and E.M. Lifschitz, Quantum Mechanics (Butterworth-Heinemann, San Francisco, 2005).

[2] Here we only consider a non-relativistic system and thus ignore the question of bound states in the Dirac equation with a repulsive potential: H. Brysk and P.F. Zweifel, Phys. Rev. C 23, 968 (1981); H. Tezuka, Japan J. Indust. App. Math, 14, 39 (1997).

[3] K. Winker, G. Thalhammer, F. Lang, R. Grimm, J. Hecker Denschlag, A.J. Daley, A. Kantian, H.P. Buchler, and P. Zoller, Nature (London) 441, 853 (2006).

[4] L. Fallani and M. Inguscio, Nature (London) 441, 820 (2006).

[5] S.M. Mahajan and A. Thyagaraja, J. Phys. A 39, L667 (2006).

[6] M. Valiente and D. Petrosyan, J. Phys. B 41, 161002 (2008); M. Valiente and D. Petrosyan, J. Phys. B 42, 121001 (2009).

[7] Y.-M. Wang and J.-Q. Liang, Phys. Rev. A 81, 045601 (2010).

[8] P.Y. Yu and M. Cardona, Fundamentals of Semiconductors (Springer, New York, 1996).

[9] K-Q. Chen, X-H. Wang, and B-Y. Gu, Phys. Rev. B 62, 9919 (2000).

[10] S. Mizuno, Phys. Rev. B 65, 193302 (2002).

[11] O. Bendix, R. Fleishmann, T. Kottos, and B. Shapiro, Phys. Rev. Lett. 103, 030402 (2009).

[12] C.M. Bender and S. Boettcher, Phys. Rev. Lett. 80, 5243 (1998).

[13] C.M. Bender, G.V. Dunne, and P.N. Meisinger, Phys. Phys. Lett. A 252, 272 (1999).

[14] C.M. Bender, D.C. Brody, and H.F. Jones, Phys. Rev. Lett. 89, 270401 (2002).

[15] We consider unnormalized eigenvectors to avoid the subtleties, associated with the $\mathcal{C P} \mathcal{T}$ innerproduct [14], that are irrelevant for our result. 